\title{
Mediation and position learning in the recall of structured letter pairs'
}

Kirk H. Smith

WALTER REED ARMY INSTITUTE OF RESEARCH

\begin{abstract}
In recalling a set of 12 structured letter pairs having either MN or PQ structure (where capital letters indicate a class of specific letters), Ss produced more intrusions of the types $\mathrm{MQ}$ and $\mathrm{PN}$ than expected by chance. This result appears to be contrary to the mediation theory of Jenkins \& Palermo (1964) and is somewhat more consistent with Braine's (1963) theory of position learning.

Problem
\end{abstract}

It has been proposed that the learning of the grammatical order of words may be explained on the basis of mediated associations. In particular, Jenkins \& Palermo (1964) have argued that the four-stage stimulus equivalence and response equivalence paradigms can account for the acquisition of the grammatical structure of twounit utterances such as found in the miniature linguistic system of Esper (1925). If the utterances, $a b, c b$, and ad, are learned in that order, mediation theory assumes that a contingency between an implicit $b$ response and explicit $d$ response is learned during the acquisition of ad, because $b$ continues to occur implicitly as a response to $a$. The theory therefore predicts that the utterance cd already has some strength since the contingencies $\mathrm{c} \longrightarrow \mathrm{b}$ and $\mathrm{b}$ (implicit) $\longrightarrow \mathrm{d}$ have been learned.

Suppose, then, that the utterances $i j, k j$, and $i l$, are also learned. Mediation theory predicts that $\mathrm{kl}$ will have gained some strength. However, the learning of all six of these utterances would not be expected to lead to the strengthening of utterances such as $\mathrm{kd}$ or $\mathrm{cl}$ which in effect mix two paradigms. No links have been established to mediate the occurrence of the latter two pairs. The item $\mathrm{j}$ has acquired some strength in the presence of $\mathrm{k}$ and might be expected to occur implicitly when the speaker says $k$, but the link from implicit $j$ to $d$ has not been formed. Similarly, the link from $k$ to implicit $b$, which might also mediate $\mathrm{kd}$, has no way of forming in this situation. Mediation theory therefore predicts that mediated generalization cannot occur on the basis of position alone (cf. Braine, 1963). In fact, the conditions under which an $\mathrm{S}$ will produce novel, rule-governed utterances are completely specified by the first three stages of the equivalence paradigm.

In unpublished work, Smith (1963) constructed sets of letter pairs from two classes of letters, A and B, with A-letters occurring first in all pairs and B-letters occurring second, and presented the pairs to Ss for recall. Some of the possible $A B$ combinations were not presented. However, when the recall productions of the Ss were examined, it was found that these unpresented
$\mathrm{AB}$ combinations appeared as intrusions too frequently and reliably to be explained as random combinations. This finding is consistent with Jenkins \& Palermo's theory of mediated association, since the presented letter pairs formed an extended acquired stimulus and response equivalence paradigm.

The present experiment provides a more direct test of the adequacy of the mediation paradigm in explaining the appearance of the grammatical intrusions found by Smith. Subjects were presented letter pairs constructed from four classes of letters, $M, N, P$, and $Q$, and exposed only to $\mathrm{MN}$ and $\mathrm{PQ}$ sequences. The $\mathrm{MN}$-pairs form an extension of the set $\mathrm{ab}, \mathrm{cb}, \mathrm{ad}$... and PQ-pairs, an extension of $\mathrm{ij}, \mathrm{kj}, \mathrm{il} \ldots$ Hence, if some $\mathrm{MN}-$ and $\mathrm{PQ}$ - combinations (e. g., cd and kl) are omitted from the presented pairs and no $\mathrm{MQ}$ or PN combinations, such as $\mathrm{kd}$ or $\mathrm{cl}$, are presented, mediation theory predicts that intrusions of the types $\mathrm{MN}$ and $\mathrm{PQ}$ should appear frequently in the Ss' recall productions, but there should be no more $\mathrm{MQ}$ and $\mathrm{PN}$ intrusions than expected under the assumption that intrusions are random combinations of the presented letters.

\section{Procedure}

A group of 40 college students enrolled in an introductory psychology course who had previously participated in an experiment involving recall of words listened to a list of 180 letter pairs containing 12 different letter combinations each presented 15 times. One pair was presented every three seconds, and a onesecond pause occurred between pairs. Ss were told they would be required to recall the pairs at the end of the list. The reading of the list was followed by three minutes of written free recall. Ss were instructed to write the pairs "in any order that they occur to you." The letters used, by classes, were M: v, s, r; N: g, k, 1; P: $z, x, d$; and $Q: m, f, j$. Omitted $M N$ and PQ pairs were vg, sk, rl, zm, xf and dj.

Results 2

The mean number of pairs of all types produced was $15.60(\mathrm{SD}=4.68)$. Subjects produced a total of 345 presented pairs $(\overline{\mathrm{X}}=8.63)$ and 279 intrusions $(\overline{\mathrm{X}}=6.98)$; of the latter, 66 introduced at least one letter which had not appeared in any presented pair $(\overline{\mathrm{X}}=1.65)$. The mean number of $\mathrm{MN}-$ and $\mathrm{PQ}$-intrusions was 1.18 per $\mathrm{S}$ and of $\mathrm{MQ}$ - and $\mathrm{PN}$-intrusions, 2.85 per $\mathrm{S}$.

Two $t$-tests were used to determine whether the latter means were greater than would be expected if Ss were producing intrusions by randomly combining two letters appearing in presented pairs. Under this 
assumption, it is expected that $6 / 120(0.05)$ of all intrusions involving presented letters will be $\mathrm{MN}-$ and $\mathrm{PQ}$-intrusions and 18/120 (0.15), will be MQ- and PNintrusions (of the 132 possible combinations of two different letters used in the presented pairs, 12 combinations were actually presented, 6 form MN- and PQintrusions, 18 form MQ- and PN-intrusions, and 96 other combinations are possible). The mean differences between the obtained and the expected number of intrusions were $0.91(t=5.45)$ for $M N-$ and $P Q$-intrusions and $2.05(\mathrm{t}=4.86)$ for $\mathrm{MQ}$ - and $\mathrm{PN}$-intrusions. With 39 degrees of freedom, both values are significantly greater than zero at the .001 level. Hence, both kinds of intrusions were more frequent than could be expected from a random combinations hypothesis.

Since the potential MQ- and PN-intrusions outnumber the potential $\mathrm{MN}$ - and $\mathrm{PQ}$-intrusions 18 to 6 , it seemed appropriate to compare the number of $\mathrm{MQ}-$ and $\mathrm{PN}-$ intrusions with three times the number of $\mathrm{MN}-$ and $\mathrm{PQ}$-intrusions. The obtained difference was not significant $(\bar{D}=0.68, t=1.53)$, indicating that the $M N-$ and $\mathrm{PQ}$-intrusions were no more likely to appear than $\mathrm{MQ}-$ and PN-intrusions.

\section{Discussion}

There appears to be no basis for an explanation of the intrusion data reported here in terms of a strictly interpreted, paradigmatic mediation theory. Although presented with pairs having the structure $M N$ or $P Q$, Ss showed by the high frequency of certain classes of intrusions that they had learned a grammar XY such that the $\mathrm{X}$-letters include letters from both $\mathbf{M}$ and $\mathbf{P}$ classes and $\mathrm{Y}$-letters include letters from both $\mathrm{N}$ and $\mathrm{Q}$ classes. In this view, $\mathrm{MN}-, \mathrm{PQ}-, \mathrm{MQ}-$, and $\mathrm{PN}-$ instrusions are all XY-intrusions. The results suggest that a more adequate account of the learning of structured letter pairs is given by Braine's theory of position learning, which predicts that Ss will learn which letters come first and which second. Since none of the letters have a higher relative frequency of appearance than any other letters, none of them can effectively form markers analogous to "function" morphemes. Braine's theory predicts that Ss will therefore learn only the absolute position of each letter (i. e., position in relation to the space, which is the only symbol which approximates a marker or "function" morpheme in this material) and not the finer-grained structure specifying the position of the class $\mathrm{N}$ letters following class $\mathrm{M}$ letters.

It should be noted that the experiments which Braine presented to support his theory of contextual generalization are also difficult to explain in terms of Jenkins \& Palermo's mediation theory. 3 Subjects were exposed to the combinations, ab, cb, ad and cd and were then required to choose between a and $d$ as a completion of the utterance $x$. The mediation paradigm supplies no basis for explaining $\mathrm{Ss}^{\prime}$ tendency to choose $\mathrm{d}$ in this instance, since the combination $\mathrm{xb}$ was not exposed. The present study may therefore be seen as a corroboration of the finding that contextual generalization can occur in cases where mediated generalization would not be expected to occur.

Referemees

BRAINE, M. D. S. On learning the grammatical order of words. Psychol. Rev., 1963, 70, 323-348.

ESPER, E. A. A technique for the experimental investigation of associative interference in artificial linguistic material. Language Monogr., 1925, No. 1 .

JENKINS, J. J., \& PALERMO, D. S. Mediation processes and the acquisition of linguistic structure. In U. Bellugi \& R. Brown (Eds.), The acquisition of language. Monogr. Soc. Res. Child Develpm., 1964, 29, No. 1 (Serial No. 92). Pp. 141-169.

SMITH, K. H. Recall of paired verbal units under various conditions of organization. Unpublished doctoral dissertation, University of Minnesota, 1963.

Notes

1. The research reported here was conducted while the author was a research fellow at the University of Minnesota and supported by National Science Foundation Grant G. 18690 to James J. Jenkins. The author has profited from conversations with Charles Clifton, Jr., concerning the design of the investigation.

2. All values reported here are exclusive of one illegible pair and 38 pairs which merely repeated a letter-combination already appearing on S's response sheet.

3. The writer is indebted to Dr. Braine for calling this to his attention. 